\title{
Shape Reconstruction of Three-Dimensional Conducting Objects Via Near-Field Measurements
}

\author{
Aslan Etminan and Levent Gürel \\ Bilkent University Computational Electromagnetics Research Center (BiLCEM), Ankara, TR-06800, Turkey \\ lgurel@gmail.com
}

\begin{abstract}
A general framework for the shape reconstruction of conducting objects is presented with the Newton minimization approach. Using a fully numerical method, the initial-guess object is evolved to reconstruct the target. The object is modeled by triangles such that the vertices are the unknowns of the inversescattering problem. The cost function is minimized as the evolving object converges to the actual target in merely tens of iterations.
\end{abstract}

\section{INTRODUCTION}

Solution of inverse-scattering problems are needed in a wide range of engineering and biomedical applications. The main goal is to obtain the geometrical and/or constitutive properties of a scattering object. Scattered fields collected from multiple receivers around the scattering object are used as the available input data set of the inversion algorithms. Furthermore, plenty of inversion algorithms have been utilized to obtain desired information about the scattering objects by using the collected data. While some studies in the inverse scattering area focus on investigating new algorithms, other studies aim at improving existing methods to increase the efficiency of the algorithms, decrease the solution time, and acquire more accurate results. The distorted Born iterative method (DBIM) is commonly used to find the distribution of the permittivity and conductivity of two-dimensional (2-D) [1] and three-dimensional (3-D) [2] dielectric objects. In DBIM, a forward solver is necessary in each iteration. A fast and efficient forward solver in inverse problems is implemented by the conjugate gradient method and the fast Fourier transform method [3]. Due to the fact that solving a forward problem is a time-consuming process, 3-D problems are rarely solved. Some works reduce a 3-D inverse problem to a 2-D one by neglecting the variations in one of the dimensions, and employing a forward solver based on the LU decomposition [4]. Inverse-scattering problems can be solved by transforming the problem to a minimization problem and utilizing an appropriate optimization technique, such as the Gauss-Newton method [5] or the steepest-descent method [6] to obtain the desired information. In this paper, we apply the steepest-descent method to solve the inversescattering problem and we successfully obtain the geometrical properties (i.e., shape and location) of a 3-D perfect electric conductor (PEC) object. The forward solver that we use in each iteration employs the multilevel fast multipole algorithm (MLFMA) [7], a powerful and accurate tool for iterative solutions of electromagnetic problems.

\section{Formulation}

To determine the shape of an unknown target, one suitable idea is to choose an appropriate initial-guess object and evolve this object in such a way that we ultimately reach the shape of the target. To this end, we should collect two sets of measurements, one from the target and the other one from the evolving object. In this work, our measurements are obtained by illuminating the object and calculating the scattered fields. After gathering the data, we use the Newton minimization approach presented in [5], [6] to evolve the initial-guess object such that the mismatch between the two sets of measurements is sufficiently reduced. We minimize the cost function, which is defined as the total mismatch between these two data sets and can be written as

$$
C(\boldsymbol{x})=\sum_{i=1}^{M}\left|S_{i}(\boldsymbol{x})-m_{i}\right|^{2},
$$

where $S_{i}(\boldsymbol{x})$ and $m_{i}$ are the $i$ th collected data from the evolving object and the target, respectively. The vector $\boldsymbol{x}$ is the modeling vector that contains the location of nodes, which model the surface of the evolving object.

\section{A. Updating the Modeling Vector}

To minimize the cost function, we use the formulation presented in [6], which is based on the steepest-descent method, to update the modeling vector, and consequently evolve the initial-guess object in such a way that the cost function tends to decrease at each iteration. Therefore, the step vector that updates the modeling vector can be chosen in the form of

$$
\boldsymbol{p}_{k}=-\frac{\left|\boldsymbol{g}_{k}\right|^{2}}{\boldsymbol{g}_{k}^{T} \cdot \overline{\boldsymbol{H}}\left(\boldsymbol{x}_{k}\right) \cdot \boldsymbol{g}_{k}} \boldsymbol{g}_{k},
$$

where $\boldsymbol{g}_{k}=\nabla C\left(\boldsymbol{x}_{k}\right)=\operatorname{Re}\left\{\overline{\boldsymbol{J}}^{H}\left(\boldsymbol{x}_{k}\right) \cdot \boldsymbol{e}(\boldsymbol{x})\right\}$ is the gradient of the cost function, $\overline{\boldsymbol{H}}\left(\boldsymbol{x}_{k}\right)=\nabla \nabla C\left(\boldsymbol{x}_{k}\right)=\operatorname{Re}\left\{\overline{\boldsymbol{J}}^{H}\left(\boldsymbol{x}_{k}\right)\right.$. $\left.\overline{\boldsymbol{J}}\left(\boldsymbol{x}_{k}\right)\right\}$ is the Hessian matrix of the cost function at the $k$ th iteration, and $\overline{\boldsymbol{J}}$ is the Jacobian matrix.

\section{B. Calculating the Jacobian Matrix}

To obtain the gradient vector $\boldsymbol{g}_{k}$ and the Hessian matrix $\bar{H}(\boldsymbol{x})$, we must first calculate the Jacobian matrix. A fully numerical and highly accurate method is presented in [6] to calculate the elements of the Jacobian matrix. To reduce the computing time of the Jacobian matrix, one possible solution is to parallelize this process. That way, $p$-fold time reduction can be obtained by distributing the computations to $p$ processors. 


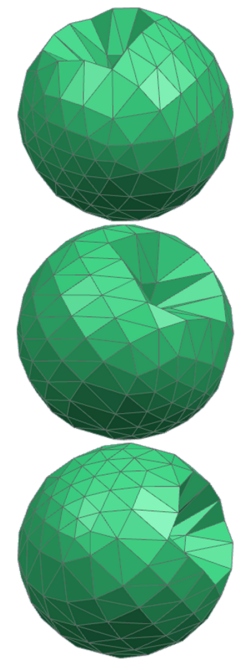

(a)

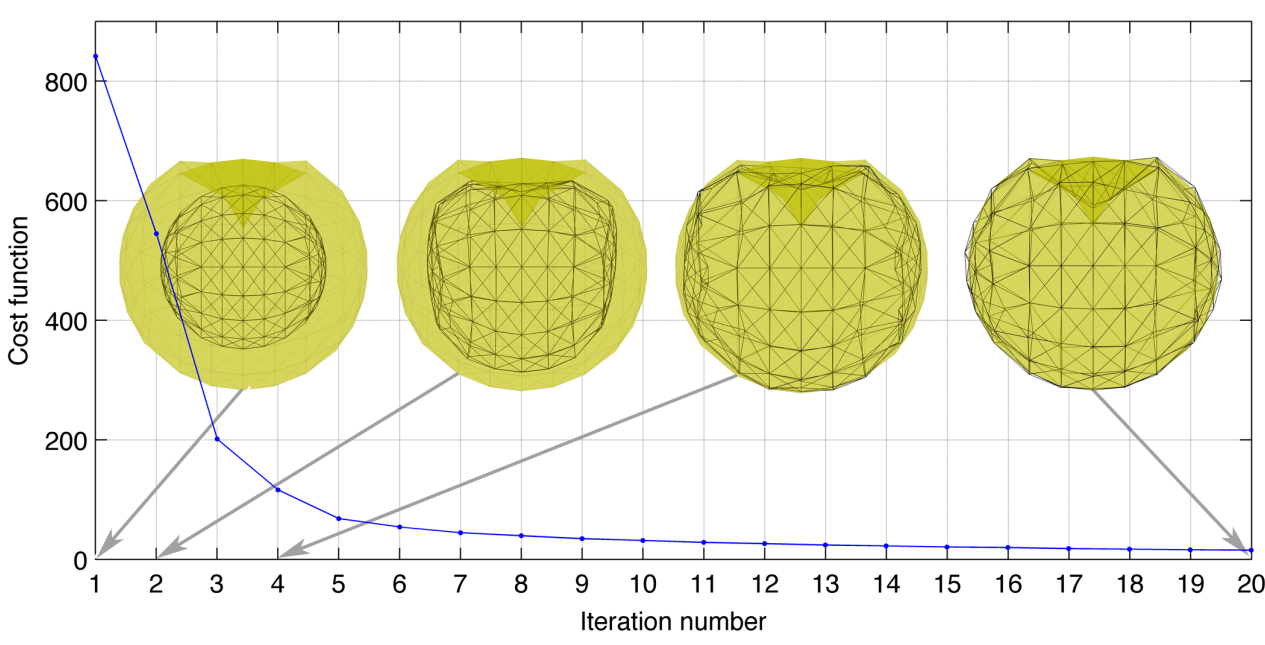

(b)

Fig. 1. Reconstruction of a concave object at $10 \mathrm{GHz}$. (a) Different views of the target object. (b) The value of the cost function in the first 20 iterations (the blue curve), the evolving object (transparent mesh), and the target (yellow object) in the first, second, fourth, and twentieth iterations.

\section{NUMERICAL RESULTS}

In this section, we present the results of reconstructing the shape of a conducting target with a complex geometry. The average radius of this target is $15 \mathrm{~mm}$, with a large concavity in the $z$-direction. To detect the shape of this target, we start with a sphere of radius $10 \mathrm{~mm}$. In our previous experiments [6], we set the transmitting and receiving antennas to be in the far-field region. In this experiment, to better simulate reallife inverse-scattering problems, we place the transmitting and receiving antennas in the near-field region. We illuminate the objects by 12 horizontal and vertical dipoles located at the $\pm x, \pm y$, and $\pm z$ axes $30 \mathrm{~mm}$ from the origin. We measure all three components of the scattered electric fields $E_{r}^{s}, E_{\theta}^{s}$, and $E_{\phi}^{s}$ from 26 different directions at $30 \mathrm{~mm}$ distance from the origin; thus the number of measurements per each incident field is $26 \times 3=78$. The operating frequency is $10 \mathrm{GHz}$ in this experiment.

It is evident in Fig. 1 that the general shape of the target is obtained in the first few iterations and the cost function is reduced to about $9 \%$ of its initial value in the first five iterations. In the next 10-15 iterations, the concavity of the target is completely detected and the cost function is finally decreased to $2.7 \%$ of its initial value. To compute the Jacobian matrix of the evolving object, which has 156 nodes, we need the solution of 156 forward problems. The solution time of each forward problem is 15.5 seconds for 12 different incident fields. Therefore, the duration of each iteration is about 2418 seconds $(156 \times 15.5)$. If we parallelize the solution of the forward problems with, for example, two and four processors, the time decreases to about 1240 and 630 seconds, resulting in a significant time reduction in each iteration.

\section{CONCLUSION}

This paper presents a shape reconstruction method for 3-D PEC objects. The shape of an unknown target is obtained by employing the Newton minimization formulation. Because computing the Jacobian matrix consumes a significant amount of time, we parallelize its calculation and achieve a remarkable reduction in the computation time.

\section{ACKNOWLEDGEMENT}

This work was supported by the Scientific and Technical Research Council of Turkey (TUBITAK) under Research Grant 111E203, by Schlumberger-Doll Research (SDR), and by contracts from ASELSAN, Turkish Aerospace Industries (TAI), and the Undersecretariat for Defense Industries (SSM).

\section{REFERENCES}

[1] T. J. Cui, W. C. Chew, A. A. Aydiner, and S. Chen, "Inverse scattering of two-dimensional dielectric objects buried in a lossy earth using the distorted Born iterative method," IEEE Geosci. Remote Sens. Lett., vol. 39, no. 2, pp. 339-346, 2001.

[2] F. Li, Q. H. Liu, and L. Song, "Three-dimensional reconstruction of objects buried in layered media using Born and distorted Born iterative methods," IEEE Geosci. Remote Sens. Lett., vol. 1, no. 2, pp. 107-111, 2004.

[3] T. Sarkar, E. Arvas, and S. Rao, "Application of FFT and the conjugate gradient method for the solution of electromagnetic radiation from electrically large and small conducting bodies," IEEE Trans. Antennas Propagat., vol. 34, no. 5, pp. 635-640, 1986.

[4] A. Abubakar, T. M. Habashy, V. L. Druskin, L. Knizhnerman, and D. Alumbaugh, "A $2.5 \mathrm{D}$ forward and inverse modeling for interpreting low-frequency electromagnetic measurements," Geophysics, vol. 73, no. 4, pp. F165-F177, Aug. 2008.

[5] T. M. Habashy and A. Abubakar, "A general framework for constraint minimization for the inversion of electromagnetic measurements," Prog. Electromagn. Res., vol. 46, pp. 265-312, 2004.

[6] A. Etminan and L. Gürel, "Microwave imaging of three-dimensional conducting objects using the Newton minimization approach," in Proc. Computational Electromagnetics Workshop, CEM'13, pp. 45-47, Izmir, Turkey, August 2013.

[7] L. Gürel and Ö. Ergül, "Hierarchical parallelization of the multilevel fast multipole algorithm (MLFMA)," Proc. IEEE, vol. 101, pp. 332 $341,2013$. 